\title{
Physical, Mechanical and Thermal properties of sand reinforced polyester resin composite
}

\author{
Md. Mahfujul Islam ${ }^{1 a^{*}}$, Md Alamgir kabir $^{1 \mathrm{~b}}$, Humayun Kabir ${ }^{1 \mathrm{c}}$, \\ Dr Farid Ahmed ${ }^{1 \mathrm{~d}}$, Md. Abdul Gafur ${ }^{1 \mathrm{e}}$ \\ $1 a^{*}$ Lecturer, Department of Natural sciences, Daffodil international University, Dhanmondi, \\ Dhaka-1205, Bangladesh. \\ ${ }^{1 b}$ Lecturer, Department of Physics, Jahangirnagar Univerity, Savar, Dhaka-1342,Bangladesh. \\ ${ }^{1 \mathrm{1c}}$ Assistant Professor, Department of Physics, Jahangirnagar Univerity, Savar, \\ Dhaka-1342,Bangladesh. \\ ${ }^{1 d}$ Professor, Department of Physics, Jahangirnagar University, Savar, Dhaka-1342, Bangladesh. \\ ${ }^{1 e}$ Senior Scientific Officer, Pilot Plant \& Process Development Center of Bangladesh Council of \\ Scientific \& Industrial Research (BCSIR), Dhaka-1205, Bangladesh \\ "Corresponding author Email: mahfujul.ns@diu.edu.bd
}

Keywords: Reinforced, polyester resin, sand, composite, elastic deformation, bulk density, Flexural strength, hardness.

\begin{abstract}
Sand reinforced polyester composite have been prepared by compression molding method with a variety of range $(0,15,30,4560 \%)$ sand respect to the unsaturated polyester resin. various parameter like mechanical, Physical and thermal properties of sand reinforced, polyester resin composite have been determined by various technique like water compressive strength ,flexural strength, hardness and thermal analysis. Physical properties like density and water intake increases with the addition of sand,compressive strength increases but flexure strength decreases with the amount of sand, on hardness test, Vickers hardness increases but on leeb rebound it decreases with amount of sand, and finally thermal conductivity decreases with the addition of sand.
\end{abstract}

\section{INTRODUCTION}

A "composite" is when two or more different materials are combined together to create a superior and unique material ${ }^{1}$. From the ancient times some common scenario of composites which attract people to use it. The advantage of composite materials over conventional materials stem largely from their higher specific strength, environmental protection factors, lightweight, elastic, corrosion and chemical resistance, stiffness and fatigue characteristics. This enables structural design to be more versatile. Composites are materials that comprise strong load carrying material known as reinforcement imbedded in weaker material known as matrix. The function of the matrix is to bond the fibers together and to transfer loads between them. in this research we used Polyester resins that are thermosetting and, as with other resins, cure exothermically. The use of excessive catalyst can, therefore, cause charring or even ignition during the curing process ${ }^{2}$.so finally unsaturated polyester resin was preferred due to their good range of mechanical properties, relatively low cost, corrosion resistance and low molecular weight. The intention of this research work is to achieve a composite with high mechanical, Physical and thermal properties using Sand with different compositions as reinforcement with polyester resin as a polymer matrix. 


\section{MATERIALS AND METHODS:}

\section{A. RAW MATERIALS:}

Raw materials that have been used in this research work were polyester resin, Sand and Methyl ethyl ketone per oxide as hardener.

\section{B. REQUIRED EQUIPMENTS FOR SAMPLE PREPARATION:}

The equipment's that have been used were oven, open mold, close mold, Poul-Otto Weber press machine, Universal testing machine, electric balance etc.

Some methods like compression molding, sieve analysis or (gradation test) used to prepare my sample, compression molding is one of them

Compression molding is a high-volume, high-pressure method suitable for molding complex, high-strength fiberglass reinforcements. ${ }^{3}$

\section{PREPARATION OF COMPOSITE}

The different percentage of Sand and polyester resin were taken to prepare composite, the first and foremost material were used here is Sand and Unsaturated polyester resin composite. Firstly sand washed properly for a long time thus several waste washed away and then dried at $100^{\circ} \mathrm{c}$ for 24 hours in a preheated oven. After drying it properly a sieve analysis or gradation test was taken. Thus we can take the sample of sand grain as down to a minimum size depending on the exact method. Being such a simple technique of particle sizing, it is probably the most common. I used $125-250 \mu$ sand grain for my research experiment. Sand was taken from 0 to $60 \mathrm{wt} \%$. The table- 1 shows different percentages of samples. A bowl was taken to mix up the raw materials of the composite. Definite amount of various percentages of sand \& polyester resin has been weighed in the bowl and then raw materials were mixed very carefully with a stirrer for about 30 Minutes. Ethyl methyl ketone peroxide was used as a hardener, as an amount of $2 \mathrm{wt} \%$ of polyester resin and weight. The mixer was then poured into the closed and open mold and was kept it 4-5 hours for drying. After drying the composite was released from the mold with Paul-Otto Weber.Press Machine by applying pressure. Polyethylene sheet was used to smooth the sides of the composite.

Table1:Sand/Polyester composite

\begin{tabular}{|c|c|c|}
\hline Composite & Sand (\%) & Polyester resin (\%) \\
\hline APC-1 & 0 & 100 \\
\hline APC-2 & 15 & 85 \\
\hline APC-3 & 30 & 70 \\
\hline APC-4 & 45 & 55 \\
\hline APC-5 & 60 & 40 \\
\hline
\end{tabular}

D. CHARACTERIZATION: Five samples of sand polyester composites having a composition of sand to polyester in the ratio of $0: 100,15: 85,30: 70,45: 55,60: 40$ were prepared and were characterized by different standard methods. Various mechanical properties such as tensile strength, elongation at break, tensile Modulus, bending strength and bending modulus of the prepared composites were studied by Universal testing machine (model 1011UK, INSTRON Corporation) system. 


\section{RESULTS AND DISCUSSION}

\subsection{PHYSICAL CHARACTERIZATION OF SPC}

3.1.A:Bulk density measurement: The bulk density effect of variation of wt. $\%$ of Sand on the bulk density of Sand reinforced polyester composites were investigated in Fig.1 . Percent of Sand

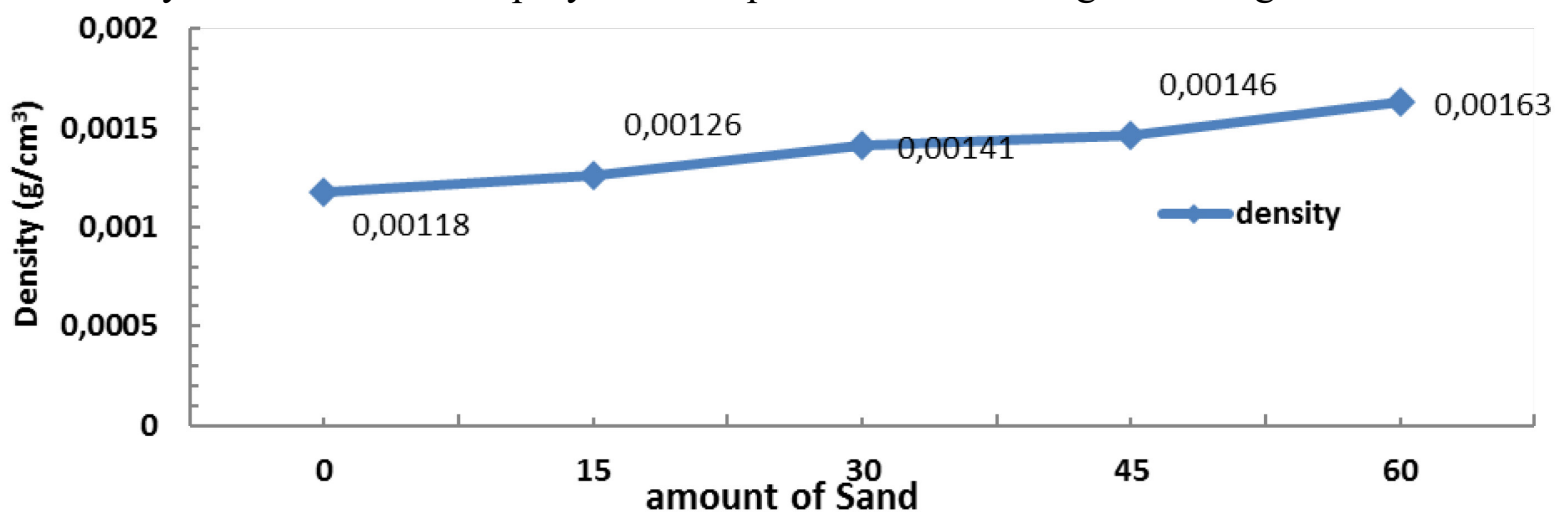

Figure 1: Effect of amount of sand on density of sand/polyester composite

$0 \%, 15 \%, 30 \%, 45 \%$, and $60 \%$ ) have been taken for fabrication. The density of Sand and polyester composites increases with the increases of the amount of Sand. Thus the bulk density increases from $0.00118 \mathrm{gm} / \mathrm{cc}$ to $0.00163 \mathrm{gm} / \mathrm{cc}$ when the amount of Sand increases from 0 to $60 \mathrm{wt} . \%$.This happened for possible smallest size of micron size in sand grain. Coat it with something that does not allow them to agglomerate, repealed each other. Micronized or pulverize it in the flow of air to make it apart. Compact them with agitation or pressure and remove air entrapped between the particles by applying vacuum.

3.1.B: WATER INTAKE: The effect of immersion time on water absorption of Sand/Polyester composites prepared with different wt $\%(0 \%, 15 \%, 30 \%, 45 \%$ and $60 \%)$ of Sand is shown in Fig. 2. It reveals that the water absorption depend on Sand content, immersion of time and amount of materials. Result shows that the water absorption increased with increasing Sand . The rate of water absorption is very low with time.

This is due to the fact that reduction in the cured polyester and the degree of cross-linking reaction, which diminishes the void spaces i.e. with the increase of molding load, the composite becomes more dense or reinforced materials are distributed properly eliminating all voids ${ }^{4}$.

Mineral fillers are hydrophobic in nature (the incapability of filler to absorb water is known to be hydrophobic filler). This is because of hydrophobic nature of sand is very small amount of water intake in the composites 5 .

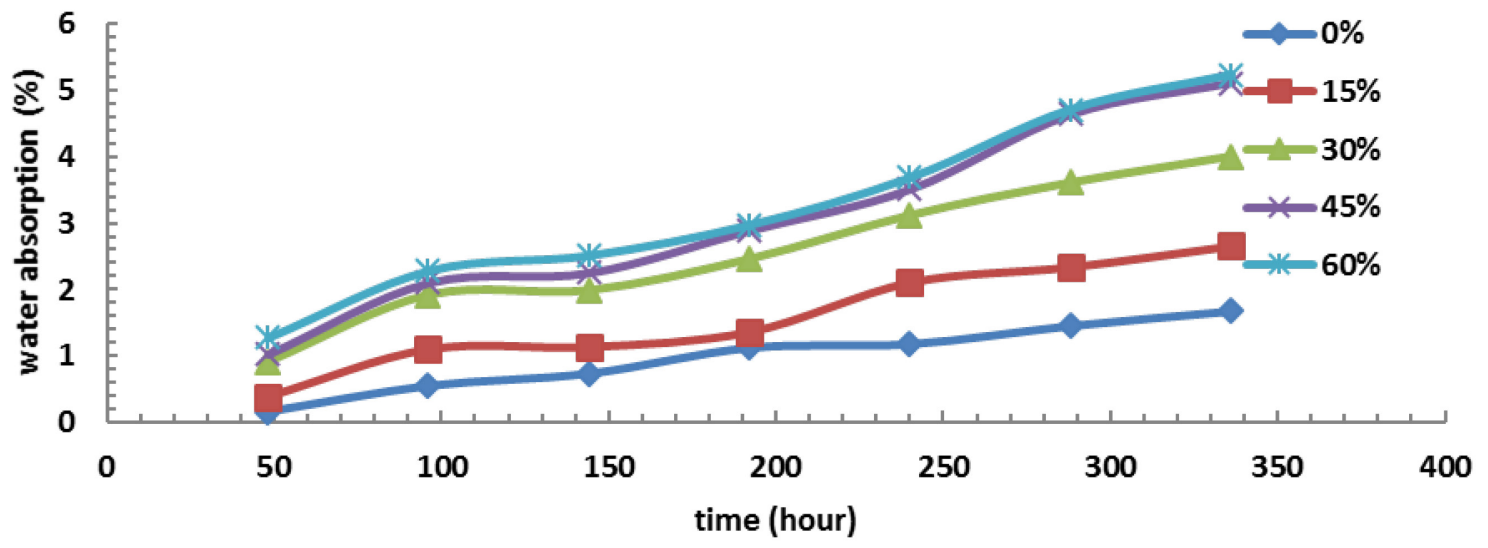

Figure 2: Effect of soaking time on water absorption property SCP composites. 


\subsection{MECHANICAL CHARACTERIZATION}

3.2. A: Elastic Modulus: Elastic modulus of SCP measured due to increase the amount of Sand on SCP, the graph shown on above, it is clearly evident from the graph that, elastic modulus increased with the amount of adding sand respectively, but suddenly the graph scale has fallen down on $45 \%$,overall we can say that elastic modulus increased due to the mixture of sand randomly.

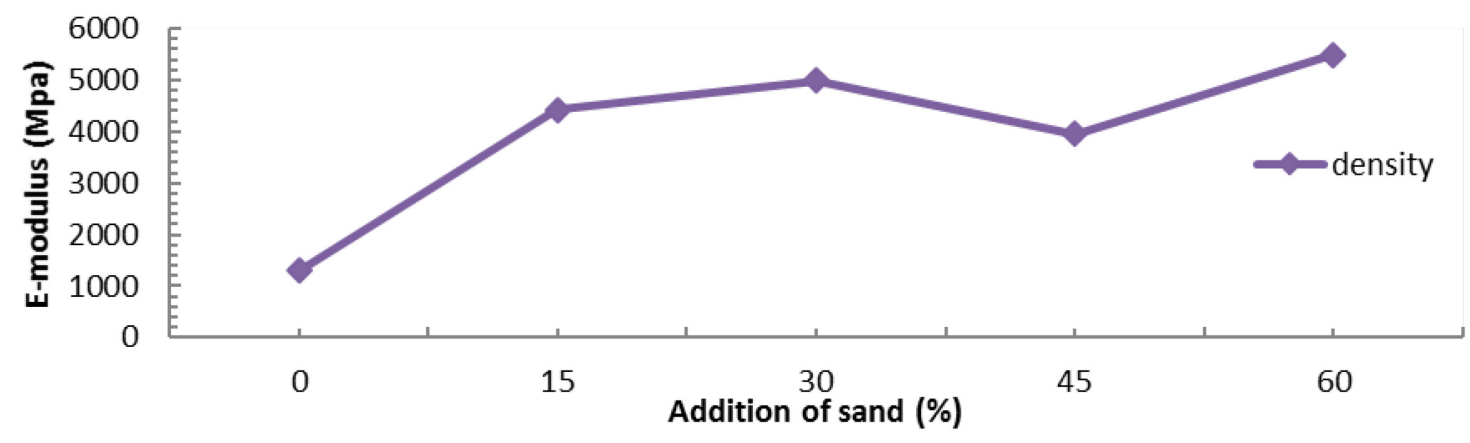

Figure 3: Effect of variation of sand on Elastic modulus of sand polyester resin composites.

\subsection{B: Flexural strength}

The flexural test was carried out by using universal testing machine or UTM (model 1011UK,INSTRON corporation). .In this experiment, the support span length was 45 and the test speed was $5 \mathrm{~mm} / \mathrm{min}$. Fig .4 illustrates the effect of addition of sand on Flexural Strength for sand/Polyester composite. It reveals that the Flexural Strength decreases with the addition of sand composites. The flexural strength of the composites is lower than polyester resins, so these composites are brittle. Shows that the increasing in volume sand promotes more interfaces and cavities formed in the composite and this can explain the decreasing of flexural strength. The decrease in strength of sand polyester composites on increasing the volume fraction of sand is due to sand being week in tension.

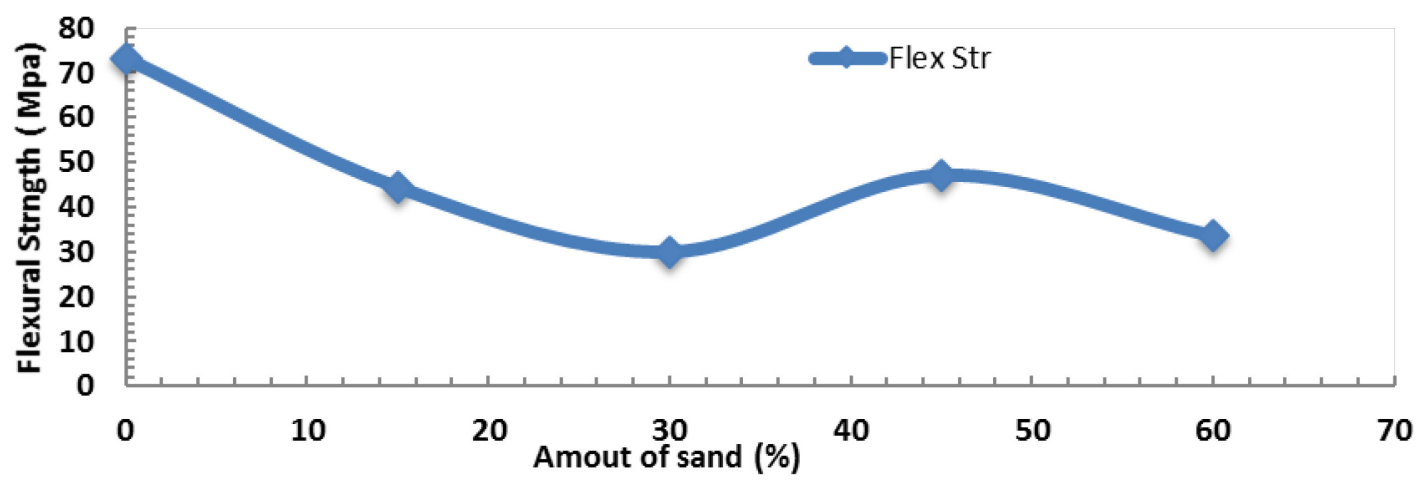

Figure 4 : Effect of amount of sand on flexural strength of sand/polyester composite

\section{THERMAL CHARACTERIZATION}

Thermal conductivities of SPCs were measured by ASTM method C201 which enables to determine thermal conductivity of bad conductors like rubber, ebonite, glass, refractory etc. In measuring the conductivity of such poor conductor, a thin layer or slab of the material is used. The effect of sand content on thermal conductivities of SPCs is shown in Fig.5. Thermal conductivity was observed to decrease from $0.00076 \mathrm{cal} / \mathrm{cm} \mathrm{sec} \operatorname{deg} \mathrm{C}$ to $0.00024 \mathrm{cal} / \mathrm{cm} \mathrm{sec} \mathrm{oC}$ with an increase in sand content from 10 to $60 \%$. The decrease in thermal conductivity with the increase of sand 
content is in agreement to the report published by Budnikov20. It is also reported that with the increases of sand content and porosity of composite the mean free path decreases resulting in lower thermal conductivity. The lower thermal conductivity of SPCs is assumed to be due to the insulating property of sand and heterogeneity in structure of composites. ${ }^{6}$

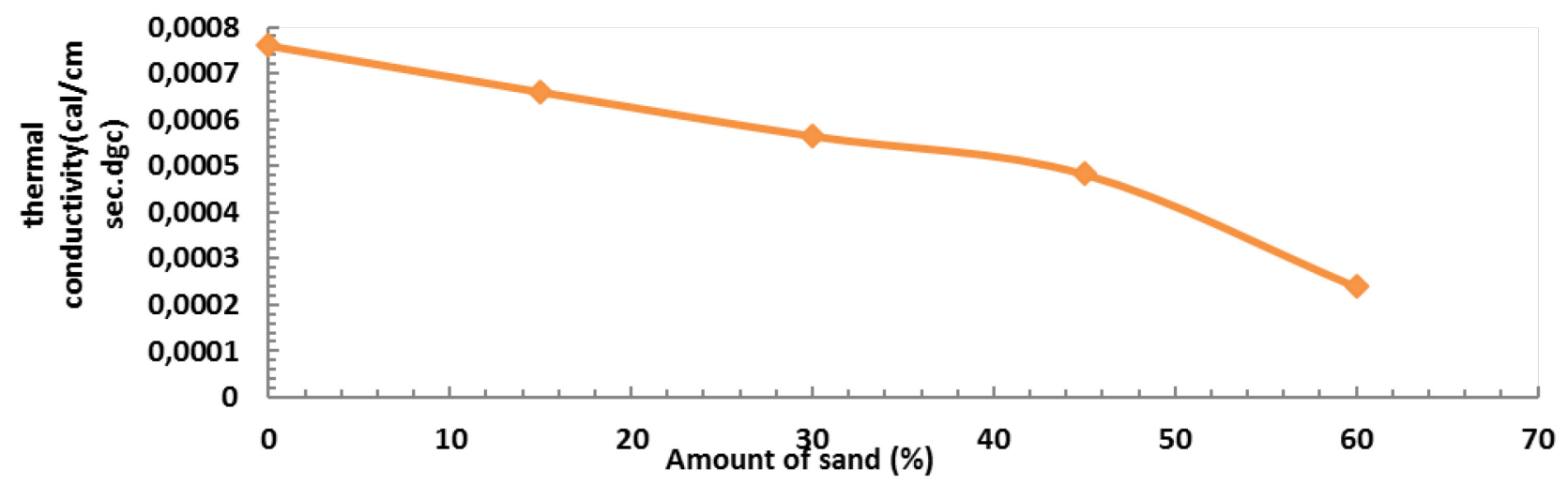

Figure 5: Effect of amount of Sand on thermal conductivity of SPCs.

\section{CONCLUSION}

Sand reinforced polyester resin composite showing promising results for different applications, It's a very effective and efficient material for engineering purpose and these has been cleared by different physical, mechanical and thermal process. Bulk density increased with the amount of an addition of sand.

For all the composites of SCP water absorption increases with increases on the addition of sand and soaking time. Flexural strength and compressive strength of SPCs decreased with an increase in sand content. The flexural strength was lower than polyester resin, so these composites are more brittle. Vickers hardness of composites increased with increasing the amount of sand content. It may be due to permanent plastic deformation. Whereas rebound hardness of the composites decreased with an increase in the addition of sand content due to elastic deformation. Thermal conductivity of the both composites decreased with an increase in sand content and it happens due to the insulating property of sand.

This wonderful properties finalize that further research can be carried out for utilization this composite product commercially.

\section{References}

[1]. http://composite.about.com/od/aboutcompositesplastics/a/What-Is-A-Composite.html

[2]. http://en.wikipedia.org/wiki/Polyester_resin

[3]. Introduction to Compression Molding". eFunda. Retrieved 19 March 2013.

[4]. Hudson N. M. The Old China Book. p. 7. ISBN 9781434477279, 1903.

[5]. Chandrasekhar S, Satyanarayana K, Pramada P and Majeed J. Effect of calcinations temperature and heating rate on the optical properties and reactivity of rice husk ash. Journal of Materials Science (Norwell), 2006; 41(1):7926-7933

[6]. Rsultana, $\mathrm{R}$ akter, z.alam, International Journal of Engineering \&Technology.IJETIJENSVol:13No.02 